\title{
Validez de una Competencia Docente con Enfoque Inclusivo en el Ámbito Universitario
}

César Delgado-Valles, Docente Investigador

Institución Benemérita y Centenaria Escuela Normal del Estado de

Chihuahua, Mexico

Rigoberto Marín-Trejo, Docente Investigador

Centro de Investigación y Docencia, Mexico

Rigoberto Marín-Uribe S., Docente Investigador

Universidad Autónoma de Chihuahua, Mexico

Doi:10.19044/esj.2020.v16n19p77～URL:http://dx.doi.org/10.19044/esj.2020.v16n19p77

\section{Resumen}

El presente artículo muestra resultados preliminares de una investigación más amplia de la Universidad Autónoma de Chihuahua, relacionada con seis competencias docentes que permite al catedrático universitario dar una respuesta oportuna al proceso de inclusión educativa, se desarrolló la validez del modelo de competencias docentes basadas en "Coordina la interacción pedagógica inclusiva" y "Gestiona la progresión de los aprendizajes" pertenecientes a una investigación más amplia de 10 competencias. Se validó el modelo propuesto por medio de la metodología Delphi, con la medición de univocidad, y pertinencia; se consolido a través del Análisis Factorial Exploratorio y Confirmatorio. La competencia tiene como propósito una docencia basada en situaciones reales que implica las formas de interacción incluyente con base a una relación educativa sustentada en la comunicación horizontal y generación de ambientes de aprendizajes con el fin de promover redes de colaboración. En el primer proceso se obtiene más de .90 en cada valor presentado en univocidad y pertinencia; bajo el Análisis Factorial Confirmatorio se agrupan en una sola competencia con los indicadores que muestra el referencial óptimo (GFI .849) y superior al valor (RMSEA .083) con las medidas de ajuste incremental y de parsimonia, superior al modelo independiente, en conclusión, es válida y confiable para el apoyo de la intervención universitaria en poblaciones con discapacidad.

Palabras clave: Competencias, discapacidad, docentes, inclusión y validación 


\title{
Validity of Teaching Competence with an Inclusive Approach in the University Environment
}

\author{
César Delgado-Valles, Docente Investigador \\ Institución Benemérita y Centenaria Escuela Normal del Estado de \\ Chihuahua, Mexico \\ Rigoberto Marín-Trejo, Docente Investigador \\ Centro de Investigación y Docencia, Mexico \\ Rigoberto Marín-Uribe S., Docente Investigador \\ Universidad Autónoma de Chihuahua, Mexico
}

\begin{abstract}
This paper focuses on the initial results of a broader Autonomous University of Chihuahua research, which is related to six teaching competencies that allow the university professor to provide timely response to the process of educational inclusion. It was developed with the validity of the educational competencies model based on "Coordinating the inclusive educational interaction" and "Managing the improvement in learning" which belongs to a broader research of 10 competencies. The model was proposed through Delphi methodology and validated through nonduality measuring and relevance. It was consolidated through Exploratory Factorial and Validating Analysis. The competency has a purpose of teaching based on real situations that involves the ways of inclusive interaction. This is done on the basis of an educational relationship that is backed up in horizontal communication and generation of learning environments in order to promote collaboration networks. In the first process, more than 0.90 was obtained in each submitted value for nonduality and relevance. Under the Validating Factorial Analysis, they were grouped together under one competency. The indicators, thus, showed the optimal referencial (GFI 0.849) and the value (RMSEA 0.083) through the measurements of incremental and parsimony adjustment, which is above the independent model. This means that it is valid and reliable for supporting university intervention in disabled populations.
\end{abstract}

Keywords: Competencies, disability, teachers, inclusion, validation

\section{Introduction}

Los gobiernos actuales, centran su atención en la política asistencial de los grupos marginados y vulnerables, haciendo visible el reconocimiento de 
las garantías individuales de esta población. A lo largo de los años ha existido una desigualdad de oportunidades en el campo educativo para los grupos marginados, el gran problema se ha vivido desde la educación básica hasta el nivel superior, en una lucha constante para la recuperación de sus derechos actuales. La política social en México, se centra en el reconocimiento de las garantías individuales de la sociedad más desprotegida, la Ley General de Educación (2019) obliga al Sistema Educativo para aceptar estudiantes con discapacidad e integrarlos y en específico garantizar su ingreso, permanencia y egreso.

Es necesario la recuperación del devenir histórico de la Educación Básica y su relación con la educación especial, como parte de las bases del fundamento teórico de la Universidad Inclusiva. En los últimos 10 años se ha incrementado notablemente el ingreso de estudiantes con discapacidad al ámbito universitario, actualmente no existe una ley que establezca la obligatoriedad de aceptarlos, pero sin duda es un derecho de las personas a ser educados y formados para su desarrollo pleno en la sociedad. La Constitución Política de los Estados Unidos Mexicanos, establece el reconocimiento de los derechos humanos, así como las garantías individuales de cada ser humano, en ellas se prohíbe todo el acto de discriminación que atente a cualquier grupo étnico, género, religión, identidad sexual o discapacidad, aún con ello existen estudiantes que cumplen con el perfil de ingreso, para cursar una carrera profesional y se quedan fuera de ella.

La interrogante sigue siendo ¿por qué de estas situaciones?, el problema es la propia desigualdad, en el momento de establecer los procesos de aprendizaje de las aulas universitarias, con las carencias de información por parte de los docentes. Sierra (2016) describe al liderazgo educativo como aquel aprendizaje profundo y duradero de dinámica, acción formativa que exige un esfuerzo constante, desarrollar habilidades para ser autogestivo en su aprendizaje, ser dinámico, creativo, abierto al cambio; pues ello contribuye a mejorar su calidad como docente, ante un reto de ofrecer educación con equidad para responder oportunamente a las exigencias de la sociedad y del sistema educativo.

En México, el ámbito universitario establece políticas educativas en favor de las poblaciones marginadas, sin embargo, las prácticas sociales, generan culturas de desintegración y deserción de los estudiantes, desde el nivel preescolar hasta al ámbito universitario, ya que exige un mayor compromiso de la sociedad en favor de esta población, sin dejar de lado propiamente al sistema educativo con una gran cantidad de deficiencias en la atención, permanencia y promoción de los estudiantes con discapacidad. La UNESCO define la educación inclusiva como el derecho de todos los alumnos a recibir una educación de calidad que satisfaga sus necesidades básicas de aprendizaje y enriquezca sus vidas (2008); ante esto no es nada nuevo, la 
Declaración Universal de los Derechos Humanos desde 1948 en su artículo 26 establece que todo ser humano tiene derecho a la educación. La universidad inclusiva, nace en México como la necesidad de cubrir la demanda educativa de una población que ha sido certificada en el nivel medio superior.

El hablar de inclusión en México, es referirse a grupos vulnerables como lo son: género, grupos religiosos, raciales y étnicos. La información, en el presente documento se enfoca únicamente a la población con diversidad funcional como eje central de la temática a investigar, se desarrolla un análisis de las corrientes actuales en relación a la inclusión de estudiantes con discapacidad en el nivel superior (Universidad Inclusiva), las competencias de los docentes, así como la validación de referenciales, bajo estos conceptos se enfocará el presente trabajo.

El concepto de inclusión hoy en día forma parte de un panorama internacional con el fin de fortalecer los sistemas educativos que eliminen todos aquellos obstaculizan la participación y el rendimiento de todos los educandos (UNESCO, 2015). En el presente trabajo, se concuerda con Brito, Porra y Ochoa (2019):

...implica abordar el tema desde un enfoque integral, considerando las necesidades de todos y todo el estudiantado, bajo una dimensión cultural y comunitaria. En este sentido, desde la educación superior, el crear culturas inclusivas, requiere necesariamente reformar la universidad de acuerdo a los nuevos cánones, donde se reconoce las desigualdades (p.160).

Por consiguiente, al momento de integrar a los estudiantes a un centro escolar se enfrentan de entrada a un fenomeno de exclusión, visto como el acto de ingreso a un sistema ajeno a su contexto de vida, Gairín y Suárez en el año 2016, definen a este proceso como "dinámico y cambiante que se configura a partir de procesos sociales, propicios de cada epoca y contexto" (p.3). Sin duda ante una educación inclusiva existe el polo opuesto al acto de exclusión, como dos variables intimamente ligadas entre sí, fenómeno que ha sido narrado a lo largo de los historia por los propios protagonistas. Sin duda la población con discapacidad, se enfrenta a diario a situaciones adeversas, ante ello se debe de consolidar la inclusión educativa como un derecho esencial para recibir una educación de calidad (Leiva \& Jiménez, 2012), con el fin de eliminar todo acto de exlusión en las escuelas.

La Convención Internacional de los Derechos de las Personas con Discapacidad del año 2006, en especifico al artículo 24, dice "reconocer los derechos de las personas con discapacidad a la educación. Con miras de hacer efectivo este derecho sin discriminación y sobre la base de la igualdad de oportunidades" (p.29), por lo tanto, el nivel de educación superior, debe 
brindar los espacios necesarios para, asegurar que los estudiantes cuenten con los elementos básicos para su permanencia en igualdad y equidad de condiciones como el resto de sus compañeros, así mismo, la agenda mundial para un desarrollo sostenible en su objetivo cuatro, establece el garantizar una educación de calidad y la promoción de oprtunidades, como una meta establecida para el año 2030, asegurando el acceso igualitario de todos los hombres y mujeres al nivel de enseñanza universitraria (PNDU, 2018).

Pérez - Castron (2016), hace referencia a las personas con discapacidad como aquel grupo, que hasta hace algunos años, estuvo practicamente excluido de la formación, y su paulatino ingreso al sistema educativo, lo cual permite la ampliación de sus derechos, al igual que de sus oportunidades, estas exigencias llevan a favorecer el ingreso, egreso y permanencia de los estudiantes con discapacidad en el ámbito universitario. Los docentes dan respuesta a la diversidad de estudiantes sin ser consciente de la funcionalidad de sus estrategias de atención, por ello existe la necesidad de establecer un marco pedagógico contribuyente a ofrecer acciones encaminadas a dar dicha respuesta. Las acciones están dirigidas a la formación de un docente con los elementos básicos en atención a la diversidad, a través de dispositivos pedagógicos que desarrollen las habilidades que permitan desarrollar programas analíticos diversificado a través del conocimiento de ajustes razonables y acciones para el trabajo cooperativo.

El termino de competencia, tiene sus origenes desde una pespectiva diversa, las primeras definiciones datan de 1997 con la propuesta de Aylett y Gregory, al hacer mención a los criterios de la función de los docentes en una educación de excelencia, su obra Criteriofor teaching competence an teaching excellence in Higher Education, establece los pirncipales dominios del docente innovador, investigador, organizado y lider en su cátedra de acuerdo a las necesidades del campo profesional, el concepto en la educación es establecido en el Proyecto Tuning Educational Struerctures in Europe (1999), de acuerdo a las directrices propuestas en dicho proyecto.

En el sentido de afinar las estructuras educativas e indentificar el intrercambio de la información para establecer metas y hacer énfasis en indicadores medibles, dinámicos acordes a las necesidades de la educación y del empleo de estás. Zabalza (2010) la define como el constructo que permite identificar dentro del campo profesional, de acuerdo a la función establecida, así como el desarrollo de habilidades para una determinada profesión, Morales y Varela (2015), la señala como un concepto claro y referido a diferentes dominios representados en el saber y descritos de la siguiente manera: sabersaber, saber-hacer, saber -ser, saber-estar y por último el saber-convivir, así mismo hace la referencia a "su eje, que son los desempeños, el saber-hacer, en este hacer se articulan los conocimientos, habilidades, actitudes y valores, que 
tienen que ser congruentes al contexto y con la complejidad del problema que se atiende para que sea un actuar responsable y efectivo"(p.38).

Por otra parte, Rogiers (2010) la define como la posibilidad de una persona de movilizar sus saberes e interiorizar el conocimiento como un conjunto integrado de recursos para la resolución de situaciones problemáticas, a lo cual, Zabalza (2010) hace referencia a tres dimensiones que configura el rol del docente: la dimensión profesional, la cual atiende la función de la caracterización del entorno laboral, las exigencias que se espera en relación a su puesto o profesión; el segundo se refiere a todo aquello relacionado aspectos de satisfacción e insatisfacción personal, motivaciones, emociones $\mathrm{y}$, por último la dimensión laboral, sistema de trabajo, contratación, promoción e incentivos, condiciones laborales, horarios de trabajo y obligaciones.

A la vez, Torra, Corral y Pérez (2012) establecen las necesidades de dar respuesta a los procesos de aprendizaje de los estudiantes de una forma más activa, mediante situaciones concretas, por tal motivo, el modelo de enseñanza del profesorado, responde a uno de los grandes retos del estudiante universitario con discapacidad, ante su proceso de inclusión en las aulas. Las universidades deben adaptarse a los tiempos actuales a fin de responder a los cambios ocurridos en las últimas décadas para lograr su fin, "la formación de profesionales que la sociedad requiere y la construcción del conocimiento" (Valenzuela, Guillén, Campa, Rodríguez \& López, 2013, p.56).

En este sentido, Zárate-Rueda, Díaz-Orozco y Ortiz-Guzmán (2017), establecen el reto de las prácticas pedagógicas del docente universitario, la carencia de formación para la atención de los estudiantes con discapacidad, así mismo la necesidad de que cada institución genere rutas de atención, la flexibilidad o adaptación del currículo a la realidad de los estudiantes, realizar ajustes razonables sin tomar medidas de discriminación o actitudes asistencialistas (Bagnato, 2017). Es decir, sean promotoras de nuevas ideas ante los retos de una educación con sentido ético y ante los nuevos tiempos formar a los docentes universitarios en materia de inclusión educativa a través de una educación de calidad (González, González, Guerrero \& Ríos, 2018).

\section{Desarrollo}

Es claro que los tiempos han rebasado a la universidad, es necesario que ésta preste atención aquellos con presentan una discapacidad, en los últimos años, la estadística de población con discapacidad, en el 2020, se ha incrementado en la Universidad Autónoma de Chihuahua, ya que cuenta con una población de 231 estudiantes de nivel pregrado y 8 de posgrado, ante está necesidad, se asume como el principal reto, la formación docente ante una transformación de modelos educativos del Sistema Universitario en México. 
Es importante generar un modelo humanista con sentido ético y fortalecer las prácticas pedagógicas desde una mirada inclusiva (UACH, 2019).

Existe la necesidad de la trasformación cultural en la sociedad para respetar al ser humano desde la perspectiva del cómo es, debido a que somos lo que hacemos y en el cómo lo hacemos, recalcar la necesidad de cambiar el rumbo y el camino trazado por los modelos educativos segregadores $\mathrm{y}$ homogenizados, ante ello existe la necesidad de construir un modelo educativo en el que prevalezca el amor, como lo más humano del ser humanoy un valor universal. Así mismo, para Casanova (2017), las sociedades democráticas son aquellas que por su naturaleza aceptan a la diversidad, vista desde el ente humano y con el valor de las aportaciones generadas sobre la misma sociedad en cualesquiera de los campos de actuación y enfoque ideológico posible, para educar ciudadanos responsables, independientes, autónomos y críticos.

El nivel universitario, aún no cuenta con bases teórico pedagógicas en la atención de estudiantes con discapacidad, sin embargo, el modelo de atención permite que exista la coyuntura para la atención de esta población y genere las condiciones para establecer un perfil referencial por parte de los docentes. El aprender a conocer, aprender a hacer, aprender a convivir y aprender a ser son las líneas de trabajo establecidas; algunos de los valores constitutivos que los estudiantes desarrollan: responsabilidad, honestidad, compromiso, creatividad, innovación, cooperación, pluralismo, liderazgo, humanismo, entre otros (Marín, 2003). Sin embargo, el referente de las competencias profesionales, es el eje central de estas estrategias, mientras que las competencias no se alteren y su consecución esté garantizada, las posibles adaptaciones de acceso al currículo (poco significativas) y de sus elementos básicos (significativas) son estrategias válidas en los estudios universitarios.

El presente trabajo, forma parte de una investigación más amplia denominada "competencias académicas del profesor universitario" que se desarrolla mediante el enfoque de métodos mixtos, en el que se aplicaron métodos cualitativos y cuantitativos. Para este trabajo se tomó en cuenta la validación de una competencia para el profesorado, desde el planteamiento inclusivo y enfoque integral, que posibilete el desarrollo profesional desde una innovación educativa, que se refleje en un proceso de inclusión en las aulas univeristarias. El Plan de Desarrollo Universitario 2016-2025 de la UACH, establece una serie de retos para trabajar en el ámbito educativo y, entre ellos la necesidad de contar con un instrumento sistémico, que permite el seguimiento de trayectorias estudiantiles, así mismo el diseño de programas que impulsen esquemas integrales para la formación y la actualización docente. El marco axiológico de esta Universidad, establece una serie de valores y principios para el trabajo de la comunidad universitaria socialmente responsable, en coherencia entre el discurso institucional y la acción educativa (UACH, 2016). 


\section{Metodología}

El estudio es de corte mixto no experimental, tipo exploratorio, la construcción del modelo siguió 3 fases para su validación: 1) Construcción del referencial; 2) Validación de competencias por juicio de expertos; 3) Validación de las competencias bajo un análisis factorial exploratorio y confirmatorio. El presente artículo muestra un informe preliminar de la validación del constructo bajo la metodología de juicio de expertos (Dephi), propuesto por Carrera (2011) y método estadístico multivariados de análisis, esta técnica se ha utilizado en los últimos años en las ciencias de humanidades y su uso se ha incrementado en el ámbito de la educación, al tratar de tener una imagen más completa de los procesos, actores y acciones del campo educativo bajo el proceso del software SPSS (Statistical Packages for Social Sciences, V.22), para el procesamiento de la información.

En la primera fase se conformó un equipo de especialistas en las áreas de diseño curricular, competencias docentes, inclusión educativa de las diferentes facultades de la universidad con el objetivo de ir definiendo el perfil docente de acuerdo a las necesidades del catedrático universitario y con ello cumplir con las áreas sustantivas de la educación superior en México; investigación, docencia y difusión, para dicha acción se construye a partir de una revisión documental de las competencias del docente en el ámbito universitario a partir del uso de matrices de comparación de los principales corrientes teóricas y dominios de acuerdo a las estrategias propuesta por Pulido (2015) donde realizó un análisis de contenido de forma eficaz al cuerpo de estudio.

\section{Juicio de expertos}

Esta fase de validación del referencial, se fundamenta en los lineamientos de Carrera (2011), a partir de ello los jueces evalúan cada una de las descripciones de los atributos de acuerdo a los siguientes apartados: 1) Descripción de las competencias mediante la univocidad (u) y la pertinencia (p); para el apoyo del ejercicio de utiliza una escala la cual toma los siguientes aspectos con sus determinados valores, en un primer momento se evaluó la univocidad; 4) El ítem es entendido o interpretado inequívocamente de una sola y única manera (óptimo), 3) El ítem es susceptible de interpretación pero puede ser entendido en general de una sola manera (elevada), 2) El ítem es susceptible de ser entendido en sentidos diversos y se encuentra más cerca de la equivocidad (baja) y por último, 1) El ítem es susceptible de no ser entendido o de ser interpretado con sentidos muy diferentes, cayendo dentro de la equivocidad (nula).

De acuerdo a la pertinencia, se toman en cuenta los mismos valores, en relación a los ítems entre sí mismos, se contempla el contexto donde se emplea: 4) El ítem es susceptible de pertenecer inequívocamente al colectivo 
docente que va dirigido (óptimo), 3) El ítem es susceptible de pertenecer mayoritariamente al colectivo docente que va dirigido (elevada), 2) El ítems es susceptible de pertenecer a diferentes colectivos (baja) y por último 1) El ítem es susceptible de no pertenecer al colectivo docente que va dirigido (nula), de acuerdo a los datos proporcionados a los expertos permitirán darle validez al instrumento en su primera versión. En esta fase, se toma en cuenta la tabla propuesta por Carrera (2011), para fines de validación de las competencias, se selecciona una escala de estimación que se describe a continuación:

Tabla 1. Criterios de univocidad y pertinencia

\begin{tabular}{clcl}
\hline \multicolumn{2}{c}{ Criterios según iU } & \multicolumn{2}{c}{ Criterios según iP } \\
\hline $\mathrm{i} \mathrm{U} \geq .80$ & $\begin{array}{l}\text { Los ítems se mantienen } \\
\text { en su forma original }\end{array}$ & $\mathrm{iP} \geq .80$ & $\begin{array}{l}\text { Los ítems se mantienen en su } \\
\text { forma original. }\end{array}$ \\
$\mathrm{i} \mathrm{U} \leq .79 \geq .60$ & $\begin{array}{l}\text { Los ítems se modifican } \\
\text { en su redacción. }\end{array}$ & $\mathrm{iP} \leq .79 \geq .60$ & $\begin{array}{l}\text { Los ítems se modifican en su } \\
\text { redacción u ubicación. }\end{array}$ \\
$\mathrm{iU} \leq .59$ & Los ítems se eliminan. & $\mathrm{iP} \leq .59$ & Los ítems se eliminan. \\
\hline
\end{tabular}

Fuente: (Carrera, Vaquero \& Balsells, 2011)

El documento fue enviado a 12 docentes universitarios con especialidad en las áreas de pedagogía, didáctica, diseño curricular, competencias docentes e inclusión educativa, con más de 10 años en servicio al frente de la docencia, el 60\% de ellos pertenece al Sistema Nacional de Investigadores del País. Una vez enviado se esperaron resultados. Con base a los criterios anteriores se obtiene los siguientes resultados en la primera revisión del modelo: se supera la puntuación del constructo con el valor de máximo de $\mathrm{iU} \geq .80$ de la univocidad del enunciado y de iP $\geq .80$ en la pertinencia, lo cual se puede observar en la siguiente tabla:

Tabla 2. Competencia "Coordina la interacción pedagógica inclusiva

\begin{tabular}{lcc}
\hline & Univocidad & Pertinencia \\
\hline $\begin{array}{l}\text { C5.1. Práctica una docencia incluyente, centrada } \\
\text { en el aprendizaje y basada en situaciones reales. }\end{array}$ & 0.95 & 1.00 \\
$\begin{array}{l}\text { C5.2. Negocia los propósitos de aprendizaje y } \\
\text { productos a lograr, considerando la diversidad y } \\
\text { bajo un enfoque inclusivo. }\end{array}$ & 0.93 & 0.98 \\
$\begin{array}{l}\text { C5.3. Aplica estrategias de intervención, para } \\
\text { lograr una interacción educativa armónica, a través } \\
\text { de procesos de comunicación horizontal y asertiva. }\end{array}$ & 0.93 & 1.00 \\
$\begin{array}{l}\text { C5.4. Genera ambientes de aprendizaje grupal, } \\
\text { para el trabajo en redes de apoyo incluyentes. }\end{array}$ & 0.95 & 1.00 \\
$\begin{array}{l}\text { C5.5. Genera la interacción entre pares en } \\
\text { ambientes y situaciones de aprendizaje diversas. }\end{array}$ & 0.98 & 0.98 \\
\hline
\end{tabular}


A su vez se recibieron sugerencias de manera cualitativa, éstas propuesta derivaron la construcción de dos enunciados más, quedando de la siguiente manera:

1. Practica una docencia incluyente centrada en el aprendizaje

2. Adecua los aprendizajes esperados y las evidencias de desempeño, considerando la diversidad y las necesidades educativas.

3. Aplica estrategias de intervención activas y participativas, que permiten la comunicación horizontal y asertiva.

4. Genera ambientes de aprendizajes grupales e incluyentes.

5. Genera la interacción entre pares académicos en ambientes y situaciones de aprendizaje diversas.

6. Atiende a la diversidad del estudiante, movilizando estrategias innovadoras y motivantes para su desarrollo.

7. Construye andamios cognitivos, para el desarrollo de las competencias en función de las necesidades de aprendizaje de los estudiantes.

8. Genera la interacción entre pares académicos en ambientes y situaciones de diversos aprendizajes.

En la otra competencia analizada también se superan el índice de valor del constructo de acuerdo a la metodología y con base a los criterios de $\mathrm{iU} \geq$ .80 en univocidad y de $\mathrm{iP} \geq .80$ en la pertinencia.

Tabla 3. Competencia "Gestiona la progresión de los aprendizajes"

\begin{tabular}{lcc}
\hline & Univocidad & Pertinencia \\
\hline $\begin{array}{l}\text { C4.1. Valora los trayectos de formación, los desempeños } \\
\text { y los niveles de logro de los estudiantes para realizar }\end{array}$ & & \\
$\begin{array}{l}\text { adecuaciones a su diseño de docencia. } \\
\text { C4.2. Adecua estrategias de aprendizaje que atiendan a }\end{array}$ & 0.95 & 1.00 \\
$\begin{array}{l}\text { las características de los estudiantes. } \\
\text { C4.3. Realiza adecuaciones curriculares que contribuye a }\end{array}$ & 0.96 & 1.00 \\
$\begin{array}{l}\text { la progresión de los aprendizajes. } \\
\text { C4.4. Retroalimenta a los estudiantes, para la } \\
\text { comprensión y solución de problemas en situaciones } \\
\text { reales de aprendizaje. }\end{array}$ & 0.90 & 0.96 \\
$\begin{array}{l}\text { C4.5. Considera los incidentes críticos que se presentan } \\
\text { en el aula para resolver situaciones de aprendizaje y } \\
\text { formación. }\end{array}$ & 0.96 & .98 \\
\hline
\end{tabular}

Por lo tanto, mediante el método Delphi, solo es necesario la realización de pequeños ajustes, para quedar en dos competencias dirigidas a los docentes al trabajar con discapacidad, se consideran las observaciones para ajuste de redacción repetida en ambas consideraciones de acuerdo a los atributos y pudiese emplearse en el mismo contexto, se propone la necesidad de reconsiderar la redacción de ambas competencias, debido al enfoque contextual y pedagógico. 


\section{Análisis Factorial Exploratorio y Confirmatorio \\ Modelo}

Es un estudio integracional a través del análisis factorial exploratorio (AFE) y confirmatorio (AFC), métodos estadísticos multivariados, este modelo estadístico destaca su importancia en el campo de la educación con la finalidad de identificar los factores e interacciones entre las características y las preferencias personales. Asimismo, para contrastar la hipótesis, la estructura de las competencias docentes se define mejor a partir de seis competencias de 10, estructura definida por un equipo de investigadores mediante un trabajo de construcción colegiada. Entre las modificaciones presentes se encuentran las dos competencias presentadas en este trabajo. Khine (2013), menciona el uso de esta técnica de investigación en el ámbito educativo. en estudios relacionados con ambientes de aprendizajes, autoeficacia, actitudes y climas organizacionales que permiten estimar el efecto entre las variables.

\section{Muestra}

El tamaño de la muestra supone un error muestral del +/- 5\% estimado para un nivel de confianza del $95 \%$ y bajo la situación más desfavorable en que la $\mathrm{p}=\mathrm{q}=0.050$. El proceso de muestreo realizado fue aleatorio estratificado. Se envió el cuestionario informatizado, por medio de la plataforma de Google $®$ formulario a un total de 975 docentes y rescatando una respuesta de 372 la cual corresponde al $38.15 \%$ de la muestra seleccionada, de las 15 Unidades Académicas de Universidad Autónoma de Chihuahua; ésto corresponde a una muestra significativa. La muestra representa un valor de importante en los Modelos de Ecuaciones Estructurales, para fines de validación del modelo se tomó como referencia la aportación de Worthington y Whittaker (2006) de acuerdo a los parámetros, señala que en función del número de constructos o ítems, cada uno de los factores debe demostrar 5 casos por parámetro de muestra adecuada (como se citó en Vargas \& Mora-Esquivel, 2017), tomando como base el criterio para la obtención de la $N$ será el resultado de multiplicación de cantidad de parámetros por el criterio respectivo del autor señalado.

\section{Instrumento}

Con base a los resultados obtenidos por medio del juicio de expertos en su segunda versión, se diseña el cuestionario a partir de la construcción colegiada, para cada una de las competencias y sus indicadores se les solicitó a los profesores que contestaran a las siguientes preguntas; ¿Qué importancia o nivel de competencia sería el deseado para los docentes? en esta primera escala, se indica la percepción sobre la importancia de que esta competencia y sus indicadores sea dominada por los profesores universitarios, en relación a 
la pertinencia se cuestionó lo siguiente; ¿Qué nivel de competencia considera tener usted actualmente?, esta escala indica la percepción sobre el grado personal de conocimientos, habilidades y dominio práctico de cada competencia y sus indicadores. Se utilizó una escala likert de acuerdo a los siguientes valores $1=$ nada $2=$ poco $3=$ regular y $4=$ mucho.

Se integra al instrumento 11 variables, correspondientes a datos generales: género, edad, grado de estudios, situación laboral y 61 variables que corresponden a los dominios de cada una de las competencias; C1. Gestiona su formación, C2. Realiza proceso de trasposición didáctica, C3. Diseña una docencia Innovadora, C4. Gestiona la progresión de los aprendizajes, C5. Coordina la interacción pedagógica inclusiva, C6. Integra recursos digitales y tecnologías en su práctica educativa, C7.Valora el desarrollo de competencias, C8. Desarrolla proceso de investigación, C9. Brinda tutorías a sus estudiantes, C10. Implementa la gestión académicoorganizativa. Se corre el Alpha de Crobanch para obtener la fiabilidad del instrumento, con valor de .97 , representando de acuerdo a George y Mallery citado por Frías (2014), donde sugiere que aquellos instrumentos con un valor superior a >.9 son excelentes, así como los ítems están inter-relacionados, por lo que se puede replicar.

\section{Procedimiento}

Los profesores fueron convocados a participar para responder el intrumento a través del Departamento de Inovacción Educativa mediante un correo invitación por medio de la plataforma electrónica de "Google formulario" $®$. Los Secretarios Académicos de cada Unidad Académica de la UACH fueron los encargados de comunicar y explicar a los docentes participantes, previa circular de la Dirección Académica en la que se especificaba todo el proceso.

\section{Resultados}

Se realizó la prueba de Kaiser-Meyer-Olkin (KMO) y Barlett, para observar si el índice KMO es mayor a .80 y tiene un nivel de significancia menor al .05, lo cual determina que el análisis factorial es pertinente:

Tabla 4. Valores de prueba KMO y Barlett Prueba de KMO y Bartlett

\begin{tabular}{llc}
\hline Medida Kaiser-Meyer-Olkin de adecuación de muestreo & .928 \\
Prueba de esfericidad de Aprox. Chi-cuadrado & 12934.4459020385 \\
Bartlett & Gl & 1830 \\
& Sig. & .000 \\
\hline
\end{tabular}

El análisis de comunalidades mostró una estructura mayor a .40, entre las conexión de las variables con el factor (1), Hoyle (2012) menciona que en este tipo de modelos surge variaciones en el diseño partiendo de la base, en 
algunos casos cambia el orden, hace mención a la utilidad como un factor de evidencia de validez de los parámetros una a uno, de manera convergente y discriminatoria a continuación se muestra en la siguiente tabla los valores identificados a partir del ejercicio de análisis determinando el efecto en el modelo nuevo:

Tabla 5. Competencia "Coordina la interacción pedagógica inclusiva"

\begin{tabular}{lc}
\hline & Factor 1 \\
\hline $\begin{array}{l}\text { 1. Practica una docencia incluyente centrada en el aprendizaje } \\
\text { 2. Adecua los aprendizajes esperados y las evidencias de desempeño, }\end{array}$ & .788 \\
considerando la diversidad y las necesidades educativas. & .768 \\
$\begin{array}{l}\text { 3. Aplica estrategias de intervención activas y participativas que } \\
\text { permiten la comunicación horizontal y asertiva. }\end{array}$ & .836 \\
$\begin{array}{l}\text { 4. Genera ambientes de aprendizajes grupal e incluyentes. } \\
\text { 5. Genera la interacción entre pares académicos en ambientes y } \\
\text { situaciones de aprendizaje diversas. }\end{array}$ & .833 \\
$\begin{array}{l}\text { 6. Atiende a la diversidad del estudiante, movilizando estrategias } \\
\text { innovadoras y motivantes para su desarrollo. }\end{array}$ & .636 \\
$\begin{array}{l}\text { 7. Construye andamios cognitivos para el desarrollo de las } \\
\text { competencias en función de las necesidades de aprendizaje de los }\end{array}$ & .775 \\
estudiantes. & .725 \\
\hline \multicolumn{2}{c}{ Tabla 6. Competencia - Gestiona la progresión de los aprendizajes } \\
\hline $\begin{array}{l}\text { 1. Adecua estrategias de aprendizaje que atiendan a las características de } \\
\text { los estudiantes. }\end{array}$ & .737 \\
2. Realiza adecuaciones curriculares que contribuye a la progresión de \\
$\begin{array}{l}\text { los aprendizajes. } \\
\text { 3. Retroalimenta a los estudiantes en la solución de problemas en } \\
\text { situaciones reales de aprendizaje. }\end{array}$ \\
$\begin{array}{l}\text { 4. Considera los incidentes críticos que se presentan en el aula para } \\
\text { resolver situaciones de aprendizaje y formación. }\end{array}$ \\
\hline
\end{tabular}

Sé analizó la varianza para identificar el número de factores que queda en el modelo jerarquizando en una sola competencia. Para la extracción del número de competencias se aplicó el criterio de Kaiser (valor exigen superior a la unidad). Se efectuó rotación (varimax) y oblicua (Oblimn) utilizando el software SPSS (Statistical Packages for Social Sciences, V.22). De acuerdo a los resultados del análisis factorial exploratorio demuestra que el modelo es viable, esta competencia se agrupa con la de "Gestiona la progresión de los aprendizajes", con base a los resultados o interceptación del ultimo muestreo, quedando de la siguiente manera, la competencia "Coordina la interacción pedagógica inclusiva" "Gestiona la progresión de los aprendizajes" el análisis factorial confirmatorio de los indicadores agrupados en un factor, la muestra del referencial es óptimo (GFI .897 y RMSEA .083) y de conformidad con las 
medidas de ajuste incremental y de parsimonia, es significativamente superior al modelo independiente :

Figura 1. Generación de la competencia

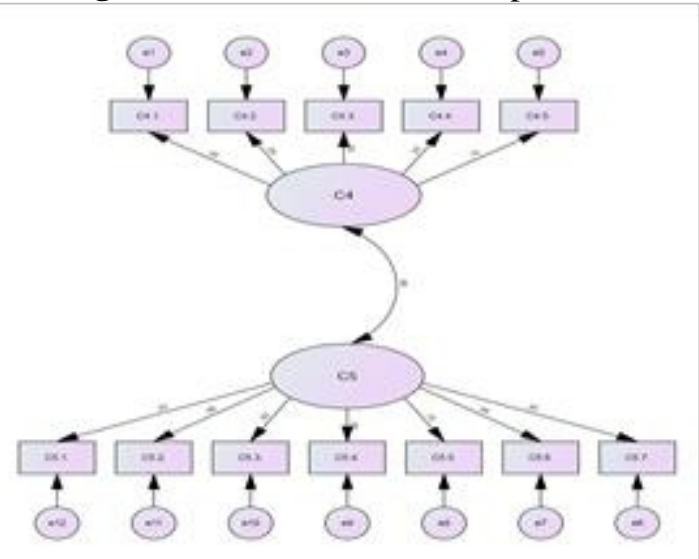

Tabla 7. Índices

\begin{tabular}{|c|c|c|c|c|c|c|c|c|}
\hline \multicolumn{5}{|c|}{ Absolutos } & \multicolumn{4}{|c|}{ Incrementales } \\
\hline$\chi^{2 \text { CMIN }}$ & GFI & RMR & RMSEA & ECVI & AGFI & TLI & NFI & CFI \\
\hline 116.12 & .897 & .013 & .083 & .949 & .849 & .958 & .940 & .966 \\
\hline \multicolumn{9}{|c|}{ Parsimonia } \\
\hline \multicolumn{9}{|c|}{ Índices de Ajuste } \\
\hline PNFI & PGFI & & $\begin{array}{l}\text { Razón Chi cu } \\
\text { normalizada }\end{array}$ & $\begin{array}{l}\text { rada } \\
/ g l) .\end{array}$ & AIC & \multirow{2}{*}{\multicolumn{3}{|c|}{$\begin{array}{c}\text { Indice de correlación } \\
\text { entre la dimensión y el } \\
\text { item. } \\
\text { OK }\end{array}$}} \\
\hline .755 & .610 & & 2.191 & & 166.12 & & & \\
\hline
\end{tabular}

Una vez que estimado el modelo es necesario evaluar la calidad, para ello se hacen ajustes de parsimonia, se valora el número de parámetros utilizados (Ruiz, Pardo \& San Martín, 2010). El análisis de factores confirmatorio permite afirmar que el modelo se agrupa en una sola competencia y en doce dominios quedando de la siguiente manera:

1. Practica una docencia incluyente centrada en el aprendizaje

2. Adecua los aprendizajes esperados y las evidencias de desempeño, considerando la diversidad y las necesidades educativas.

3. Aplica estrategias de intervención activas y participativas que permiten la comunicación horizontal y asertiva.

4. Genera ambientes de aprendizajes grupal e incluyentes.

5. Genera la interacción entre pares académicos en ambientes y situaciones de aprendizaje diversas.

6. Atiende a la diversidad del estudiante, movilizando estrategias innovadoras y motivantes para su desarrollo. 
7. Construye andamios cognitivos para el desarrollo de las competencias en función de las necesidades de aprendizaje de los estudiantes Genera la interacción entre pares académicos en ambientes y situaciones de aprendizaje diversas.

8. Valora los trayectos de formación, los desempeños y los niveles de logro de los estudiantes para realizar adecuaciones a su diseño de docencia

9. Adecua estrategias de aprendizaje que atiendan a las características de los estudiantes

10. Realiza adecuaciones curriculares que contribuye a la progresión de los aprendizajes.

11. Retroalimenta a los estudiantes para la comprensión y solución de problemas en situaciones reales de aprendizaje.

12. Considera los incidentes críticos que se presentan en el aula para resolver situaciones de aprendizaje y formación.

\section{Conclusión}

Los resultados demostraron un modelo confiable en su primera versión según los criterios de univocidad (iU). con ello se valida el contenido de los dominios o aprendizajes esperados de las 10 competencias (Carrera, Vaquero \& Balsells, 2011), asi mismo, se cuenta con un instrumento mejor estructurado. Así como se demuestra en la evaluación de la consistencia interna confiable con un Alpha de Crobanch mayor a >.9 según los criterios de Frías-Navarro (2019). Posteriomente se réplica en una población de 372 docentes con una muestra significativa, por medio de un modelo ecuaciones estructurales siguiendo el proceso del AFE y AFC; demostrando los resultados un valor de 0.928; según la prueba de Kaiser-Meyer-Olkin (KMO) y Barlett, mayor a $.80 \mathrm{y}$; una significancia menor a 0.05 , lo cual demuestra que el modelo es pertinente.

Los dominios presentaron una comunalidad mayor a .40, se ajustó el perfil al mejor modelo, lo cual muestra como resultado de ambos análisis las competencias se convirtieron en un modelo valido, es necesario establecer un perfil profesor universitario que atienda dichas demandas. Con base a los criterios del modelo evaluado se ajustó de acuerdo a los valores que presentaron una covarianza muy alta, esto permitió hacer los ajustes de los criterios exigidos, con una muestra referencial óptima en la competencia con valores de GFI y RMSEA mayores a .06 y de conformidad con las medidas de ajuste incremental y de parsimonia significativamente superior al modelo independiente.

El sistema universitario, enfrenta grandes desafios de brindar una educación de calidad a los estudiantes, una demanda social visible ante la necesidad de brindar un espacio a los grupos desprotegidos, ante un derecho 
moral, más que legal, pero esto no garantiza su permanencia y promoción dentro de la institución, el mayor reto lo enfrenta el docente ante una diversidad de estudiantes y bajo un modelo estricto en su estilo de enseñanza, con un gran desafío de formación.

La educación inclusiva, tiene el imperativo ético de garantizar el acceso, la plena participación y el aprendizaje de cada estudiante independiente de sus diferencias personales, procedencia social y cultural (Murillo \& Duk, 2016) así mismo, no se puede prescindir de los orígenes de está, como los principales movimientos orientados a la trasformación de los sistemas educativos, para responder a la diversidad y hacer efectivos los derechos de igualdad, como la principal meta sería determinar los apoyos necesarios para que los estudiantes, puedan realizar sus estudios de manera equitatativa en relación al resto de la población (Corrales, Soto \& Villafañe, 2016).

Ante las necesidades latentes de un docente formado en una educación inclusiva, es válido el modelo de competencias para su aplicación como la necesidad prioritaria ante los momentos actuales que vive el ámbito universitario y de acuerdo a la necesidad de formar a sus docentes para responder a las necesidades de los estudiantes que presentan discapacidad, desarrollo de competencias desde un enfoque de inclusión como las herramientas de apoyo en el diseño de programas analíticos diversificados.

De acuerdo a la información revisada, se asume al termino como el proceso de respuesta ante la necesidad de un modelo educativo y el acercamiento a las prácticas educativas en la formación del docente, con relación al perfil deseable, de acuerdo a los recursos cognitivos, metacognitivos, y éticos; en este último se ubica la necesidad de dicho proyecto de investigación, con el fin de responder las necesidades de la diversidad de estudiantes que se encuentran en las instituciones educativas, en específico a la población con discapacidad inscritos en la Universidad Autónoma de Chihuahua.

Los retos que implica, es la aplicación de las competencias en el ámbito universitario y visualizar el dominio ante las necesidades de atender desde los diferentes escenarios, la puesta en práctica de estas acciones deberá arrojar resultados positivos, en relación a la necesidad actual de un grupo de estudiantes, en condiciones adversas ante un sistema homogéneo, lo que llevará a desarrollar prácticas inclusivas de beneficio, no solo para ellos, sino para el grupo en general, como producto de una pedagogía de equidad e igualdad de oportunidades, el docente universitario diseñará actividades diversificadas que impulsen desde una óptica al resto del grupo, el desafío será la consolidación de un sistema universitario, que se adecue a las necesidades de sus estudiantes. 
Lo más trascendente, es informar a la sociedad sobre la importancia de generar espacios inclusivos, no solo en el ámbito escolar, la siguiente meta es crear las condiciones para el campo laboral, no se debe permitir que sigan existiendo estás prácticas de discriminación, por ello la tarea es seguir trabajando en esta condición humana de la sociedad (González, González, Guerrero \& Ríos, 2018).

\section{References:}

1. Bagnato, M. J. (2017). La inclusión educativa en la enseñanza superior: retos y demandas, 15-26.

2. Brito, S., Basualto, L. P., \& Reyes, L. O. (2019). Inclusión Social/Educativa, en Clave de Educación. Revista Latinoamericana de Educación Inclusiva, 157-172.

3. Carrera, F. F., Vaquero, E. T., \& Balsells, M. B. (2011). Instrumento de evaluación de competencias digitales para adolescentes en riesgo. Revista Electrónica de Tecnología Educativa, 35, 1-25.

4. Casanova, M. A. (2017). Educación inclusiva en las aulas. España: Arco.

5. Corrales, H. A., Soto, H. V., \& Villafañe, H. G. (2016). Barreras de aprendizaje para estudiantes con discpacidad en unaUniversidad Chilena. Revista Electrónica "Actualidades Investigativas en Educación", 1-29.

6. CNDH (2006). La convención sobre los derechos de las personas con discapacidad. Asamblea General de las Naciones Unidas.

7. DOF (2019). Ley General de Educación. Recuperado el 29 de junio de 2020 , de

http://www.diputados.gob.mx/LeyesBiblio/pdf/LGE_300919.pdf

8. Espacio Europeo de Educación Superior (1999). Declaración de Bolonia. Recuperado de http://eees.umh.es/contenidos/Documentos/ DeclaracionBolonia.pdf

9. Frías-Navarro, D. (2019). Apuntes de consistencia interna de las puntuaciones de un instrumento de medida. Universidad de Valencia. España. Disponible en: https://www.uv.es/friasnav/AlfaCronbach.pdf

10. Gairín, S. J. \& Suárez, C. I. (2016). inclusión y grupos en situación de vulnerabilidad: Orientaciones para repensar el rol de las universidades. Revista Electrónica de Educación, 1-15.

11. González, R. Y., González, B. S., Guerrero, V. D., \& Ríos, G. P. (2018). Principales Retos a la Inclusión en la Educación Superior Ecuatoriana. Enfermería Investiga, Investigación, Vinculación, Docencia y Gestión, 3, 84-90. 
12. González-González, D., Gutiérrez-Barroso, J., González-Herrera, A. I., \& Márquez-Domínguez, Y. (2017). Inserción Laboral De Personas Con Discapacidad. European Scientific Journal, 13(29), 109-124.

13. Hoyle, R. (2012). Introduction and Overview. En Rick Hoyle. Handbook of Structural Equation modeling. The Guilford Press., 3-16.

14. Khine, M. S. (2013). Structural Equations Modeling in Educational Research and Practice. (K. En Myint Swe, Ed.) USA: Boston: Sense Publishers.

15. Leiva, O. J. \& Jiménez, H. A. (2012). La educación inclusiva en la Universidad del siglo XXI: Un proceso permanente de cambio. Revista Electrónica de Investigación y Docencia (8), 41-62.

16. Marín, R. U. (2003). El Modelo Educativo de la UACH: Elementos para su. México: Universidad Autónoma de Chihuahua.

17. Morales, J. D. \& Varela, M. R. (2015). El debate en torno al concepto de competencias. 4(13), 36-41.

18. Murillo, J. \& Duk, C. (2016). Segregación Escolar e Inclusión. Revista Latinoamericana de Educación Inclusiva, 11-13.

19. Pérez-Castro, J. (2016). La inclusión de las personas con discapacidad en la educación superior en México. Revista Electrónica de Educación Sinética, 1-15.

20. PNUD (2018). Programa de las Naciones Unidas para el Desarrollo. (P. d. Desarrollo, Editor) Obtenido de

https://www.undp.org/content/undp/es/home/sustainabledevelopment-goals/goal-4-quality-education.html

21. Pulido, P. M. (2015). Ceremonial y protocolo: métodos y técnicas de investigación científic. Revista opción, 1137-1156.

22. Roegiers, X. (2010). Pedagogía de la Integración. Competencias e Integración de los Conocimientos en la Enseñanza. México.

23. Ruiz, M. A., Pardo, A., \& San Martín, R. (2010). Modelo de Ecuaciones Estructurales. Papeles del Psicólogo, 31(1), 34-45.

24. Sierra, G. M. ( 2016). Liderazgo educativo en el siglo XXI, desde la perspectiva del emprendimiento sostenible. Revista Escuela de Administración de Negocios(81), 111-128.

25. Torra, I., Corral, I., \& Pérez, J. M. (2012). Identificación de competencias docentes que oriente el desarrollo de planes de formación dirigidos a profesorado universitario. Revista de Docencia Universitaria, 21-56.

26. UACH (2019). Estadística Básica. Chihuahua: Universidad Autónoma de Chihuahua.

27. UACH (2016). Plan de Desarrollo Universitario 2016-2025. México: Universidad Autónoma de Chihuahua. 
28. UNESCO (2008). Conferencia Internacional de Educación: La Educación inclusiva, el camino hacia al futuro. Ginebra: UNESCO. Recuperado el 30 de abril de 2020, de http://www.ibe.unesco.org/fileadmin/user_upload/Policy_Dialogue/4 8th_ICE/General_Presentation-48CIE-4_Spanish_.pdf

29. UNESCO (2015). Educación 2030 "Declaración de Incheon". Korea: Organización de las Naciones Unidas para la Educación.

30. Vargas, T. H. \& Mora-Esquivel, R. (2017). Tamaño de las muestras en modelos de ecuaciones estructurales con constructos latente: Un método práctico. Revista Electrónica "Actualidades Investigativas en Educación", 17(1), 2-34.

31. Zabalza, B. M. (2010). Competencias docentes del profesorado universitario. Calidad y desarrollo profesional. España: Narcea.

32. Zárate-Rueda, R., Díaz-Orozco, S. P., \& Ortiz-Guzmán, L. (2017). Educación Superior Inclusiva: Un reto para las prácticas pedagógicas. Revista Electrónica Educare, 21(3), 1-24. 